08,04

\title{
Парамагнитный резонанс иттрий-алюминиевого граната, легированного ионами ${ }^{151} \mathrm{Eu}^{2+}$
}

\author{
() В.А. Важенин ${ }^{1}$, А.П. Потапов ${ }^{1}$, Г.Р. Асатрян ${ }^{2}$, А.Г. Петросян ${ }^{3}$, К.Л. Ованесян ${ }^{3}$, \\ А.В. Фокин ${ }^{1}$, Г.С. Шакуров ${ }^{4}$ \\ ${ }^{1}$ Институт естественных наук Уральского федерального университета, \\ Екатеринбург, Россия \\ ${ }^{2}$ Физико-технический институт им. А.Ф. Иоффе РАН, \\ Санкт-Петербург, Россия \\ ${ }^{3}$ Институт физических исследований НАН Армении, \\ Аштарак, Армения \\ ${ }^{4}$ Казанский физико-технический институт им. Е.К. Завойского КазНЦ РАН, \\ Казань, Россия \\ E-mail: Vladimir.Vazhenin@urfu.ru
}

(Поступила в Редакцию 25 мая 2016 г.)

В кристаллах иттрий-алюминиевого граната исследованы ромбические центры ${ }^{151} \mathrm{Eu}^{2+}$. С учетом положения компонент сверхтонкой структуры определены параметры начального расщепления и сверхтонкого взаимодействия. В результате анализа вида сверхтонкой структуры, сформированной разрешенными и запрещенными электронно-ядерными переходами, определены относительные знаки тонкой и сверхтонкой структур.

Работа выполнена в рамках государственного задания Минобрнауки РФ для Уральского федерального университета (проекты 2457 и 3.571.2014/К), а также поддержана грантом РФФИ № 15-52-05040 Арм_а и грантом Госкомитета по науке Армении 15RF-003.

Основные измерения проведены на спектрометре Центра коллективного пользования „Современные нанотехнологии“ Уральского федерального университета.

\section{1. Введение}

В работе [1] был исследован электронный парамагнитный резонанс (ЭПР) высокоспиновых $(S=7 / 2)$ ромбических $\left(D_{2}\right)$ центров $\mathrm{Eu}^{2+}$ и $\mathrm{Gd}^{3+}$ в кристаллах $\mathrm{Y}_{3} \mathrm{Al}_{5} \mathrm{O}_{12}$, легированных редкоземельными ионами с естественным содержанием изотопов. Были определены параметры тонкой структуры спиновых гамильтонианов указанных центров, для центров $\mathrm{Eu}^{2+}$ получено примерное равенство параметров начального расщепления $b_{20}$ и $b_{22}$. Вследствие перекрытия компонент сверхтонкой структуры (СТС) двух изотопов европия удалось лишь оценить компоненты $A$-тензора. По этой же причине в процедуре оптимизации параметров начального расщепления ионов $\mathrm{Eu}^{2+}$ использовались положения центров СТС (в случае ее наличия) или положения сигналов с неразрешенной СТС. Настоящая работа посвящена детальному исследованию и описанию в иттрий-алюминиевом гранате (YAG) ромбических центров ${ }^{151} \mathrm{Eu}^{2+}$ в единой процедуре, учитывающей как тонкую, так и сверхтонкую структуру спектра.

\section{2. Выращивание кристаллов и методика измерений}

Монокристаллы YAG: ${ }^{151} \mathrm{Eu}$ выращивались методом вертикальной направленной кристаллизации из расплава $[2,3]$ в атмосфере $\mathrm{Ar} / \mathrm{H}_{2}\left(\begin{array}{lll}10 \% & \mathrm{H}_{2}\end{array}\right)$ при нормаль- ном давлении с использованием молибденовых контейнеров диаметром $14 \mathrm{~mm}$ и затравочных кристаллов, ориентированных по кристаллографической оси [001]. В качестве исходных компонентов материалов использовались высокочистые $(99.99 \%)$ оксиды $\mathrm{Y}_{2} \mathrm{O}_{3}, \mathrm{Eu}_{2} \mathrm{O}_{3}$ и кристаллический $\mathrm{Al}_{2} \mathrm{O}_{3}$. Изотопный состав использованного оксида $\mathrm{Eu}_{2} \mathrm{O}_{3}$ соответствовал ${ }^{151} \mathrm{Eu}(97.5 \%)$ и ${ }^{153} \mathrm{Eu}(2.5 \%)$. Для стабилизации достаточно высоких концентраций $\mathrm{Eu}^{2+}$ в расплав вводились дополнительные четырехвалентные примеси в виде оксидов $\mathrm{SiO}_{2}$ или $\mathrm{HfO}_{2}$. Концентрации примесей составляли 0.2 at.\% для $\mathrm{Eu}$ и 0.2 at.\% для $\mathrm{Si}$ или Hf. В расчетах исходных составов принималось, что ионы $\mathrm{Si}^{4+}$ замещают тетраэдрические узлы $\mathrm{Al}^{3+}$, а ионы $\mathrm{Hf}^{4+}$ - додекаэдрические узлы $\mathrm{Y}^{3+}$. Процессы кристаллизации проводились при скорости $1.5 \mathrm{~mm} / \mathrm{h}$.

При указанных условиях роста были получены прозрачные кристаллы с фиолетово-синей окраской, обусловленной ионами $\mathrm{Eu}^{2+}$ [4]. Окраска плавно изменяется по длине кристаллов от фиолетовой до темно-синей, что обусловлено меньшим единицы коэффициентом распределения примесных ионов (ионные радиусы $\mathrm{Eu}^{2+}$ и $\mathrm{Y}^{3+}$ равны 1.25 и $1.019 \AA$ соответственно [5]). Дополнительные ионы $\mathrm{Hf}^{4+}$ или $\mathrm{Si}^{4+}$ по размерам меньше замещаемых ионов основы и ведут к частичной компенсации объема, тем не менее коэффициент распределения $\mathrm{Eu}^{2+}$ остается меньшим единицы. Качество полученных кристаллов контролировалось на поляризационном 
микроскопе МПС-2. Единственным макродефектом в кристаллах были гранные формы роста $\{211\}$ и $\{110\}$, которые при выбранной ориентации наблюдались лишь в периферийных областях кристаллов.

Для ЭПР исследований выбирались наиболее качественные области кристаллов, в которых содержание $\mathrm{Eu}^{2+}$ максимально. Образцы, не содержащие гранных форм роста, далее ориентировались на рентгеновском аппарате УРС-2.0 и вырезались в кристаллографических плоскостях (001) и (011) в виде прямоугольных параллелепипедов размером $1.5 \times 2 \times 5 \mathrm{~mm}$.

Измерения ЭПР проводились на спектрометре трехсантиметрового диапазона EMX Plus Bruker в интервале температур 120-300 K с использованием штатного одноосного автоматического гониометра. Для реализации вращения в двух перпендикулярных плоскостях кристалл помещался в тефлоновый бочонок, расположенный на штанге гониометра и обеспечивающий вращение образца вокруг горизонтальной оси.

\section{3. Результаты}

В структуре граната (пространственная группа Ia $3 d$ $\left.\left(O_{h}^{10}\right)\right)$ существует двенадцать структурно-эквивалентных, но различно ориентированных позиций $\mathrm{Y}^{3+} \mathrm{c}$ симметрией $D_{2}$. Именно эти позиции занимают ионы европия [1]. Спектры центров в позициях, связанных инверсией, в магнитном резонансе не отличаются, поэтому наблюдается шесть магнитно-неэквивалентных центров, демонстрирующих в ориентациях $\mathbf{B}\left\|\mathbf{C}_{2}, \mathbf{B}\right\| \mathbf{C}_{3}, \mathbf{B} \| \mathbf{C}_{4}$ $\left(\mathbf{B}-\right.$ индукция магнитного поля, $\mathbf{C}_{i}$ - оси кристалла) объединение в эквивалентные группы. Оси $\mathbf{Z}$ и $\mathbf{Y}$ локальной системы координат указанных ромбических центров, как и в работе [1], были выбраны параллельными осям $\mathbf{C}_{2}$ кубической ячейки, ось $\mathbf{X}$ совпадала с направлением $\mathbf{C}_{4}$ кристалла.

В ориентации $\mathbf{B} \| \mathbf{C}_{2}$ четыре ромбических центра (из шести наблюдаемых) эквивалентны $\left(\theta=60^{\circ}\right)$, а оставшиеся два центра являются одиночными с $\theta=0$ и 90․ На рис. 1 представлены ЭПР-спектры $(300 \mathrm{~K}$, поле $\mathbf{B}$ параллельно оси $\mathbf{C}_{2}$ элементарной ячейки) при легировании кристаллов $\mathrm{Y}_{3} \mathrm{Al}_{5} \mathrm{O}_{12}$ европием с естественным содержанием изотопов (ядерные спины ${ }^{151} \mathrm{Eu}$, ${ }^{153} \mathrm{Eu}$ равны $5 / 2$, распространенность 48 и $52 \%$ соответственно) и обогащенным ${ }^{151} \mathrm{Eu}$. Кроме очевидного увеличения разрешения в случае обогащенного ${ }^{151} \mathrm{Eu} \mathrm{в}$ глаза бросается различие степени разрешения компонент СТС переходов, принадлежащих центрам с Z $\| \mathbf{B}$ и $\mathbf{Z} \perp \mathbf{B}$. Положения остальных переходов параллельного и перпендикулярного центров $\mathrm{Eu}^{2+}$ при $\mathbf{B} \| \mathbf{C}_{2}$ (рис. 2) также попарно близки, при этом соотношение разрешения и ширины компонент СТС (рис. 3) аналогично представленному на рис. 1, хотя выражено не так ярко. Следует отметить, что уменьшение пиковой интенсивности сигналов СТС параллельного центра на рис. 3 обусловлено их большей шириной. Так же как в [1], сигналы с менее разрешенной СТС отнесены

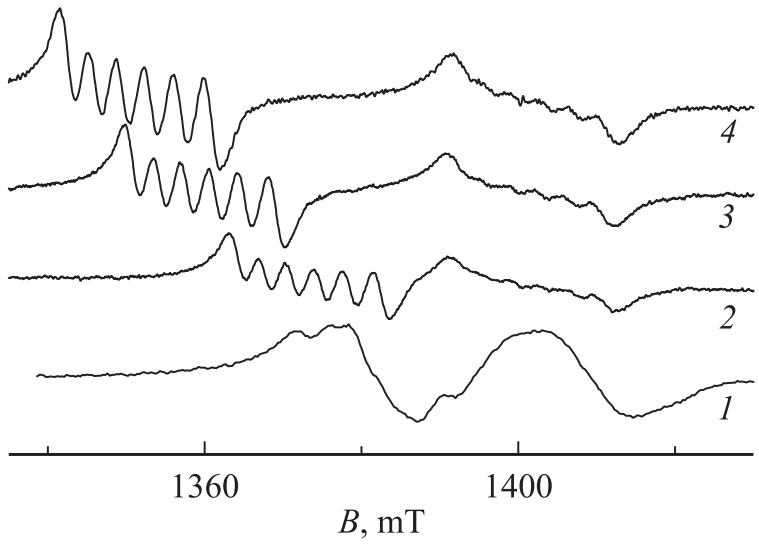

Pис. 1. Спектр ЭПР переходов $3 \leftrightarrow 4$ (B $\perp \mathbf{Z}$, нижний по полю) и $5 \leftrightarrow 6$ (B $\| \mathbf{Z}$, верхний по полю) центров $\mathrm{Eu}^{2+}$ в $\mathrm{Y}_{3} \mathrm{Al}_{5} \mathrm{O}_{12}: 0.2 \% \mathrm{Eu}, 0.2 \% \mathrm{Si}$ при $\mathbf{B} \| \mathbf{C}_{2}$. Нумерация электронных уровней энергии снизу вверх (см. рис. 2). 1 - европий с естественным содержанием изотопов на частоте $9870 \mathrm{MHz}$ при $300 \mathrm{~K}, 2-4-$ обогащенный ${ }^{151} \mathrm{Eu}$, частота $9457 \mathrm{MHz}$, температура 300, 200, $120 \mathrm{~K}$ соответственно.

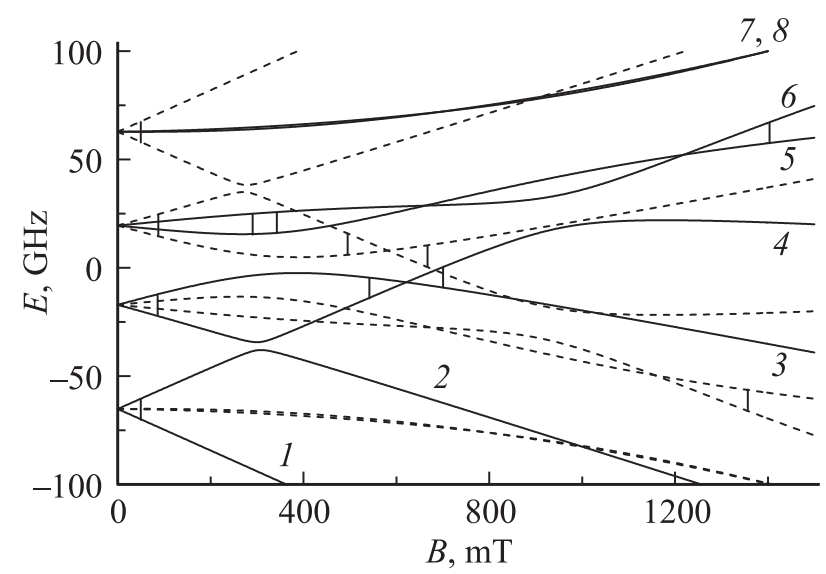

Рис. 2. Электронные уровни энергии и расчетные переходы параллельного (сплошные кривые) и перпендикулярного (штриховые) центров $\mathrm{Eu}^{2+}$ при $\mathbf{B} \| \mathbf{C}_{2}$ и $300 \mathrm{~K}$.

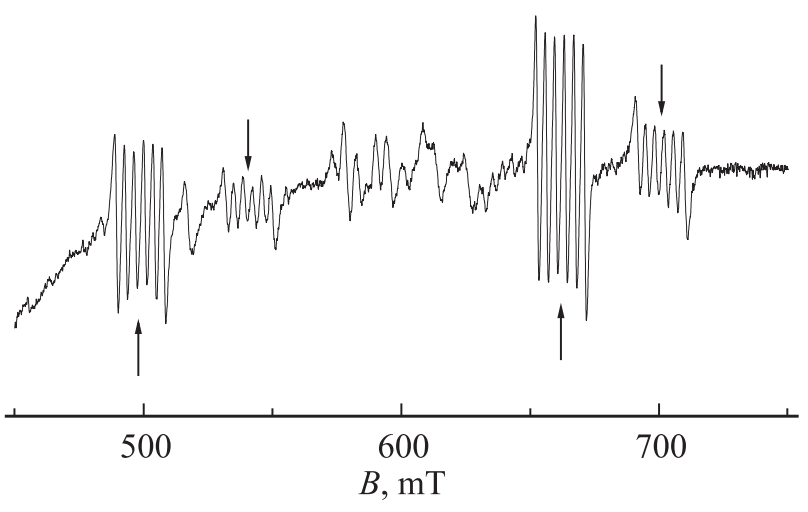

Рис. 3. Экспериментальный ЭПР-спектр переходов параллельного $(\mathbf{B} \| \mathbf{Z}$, верхние стрелки) и перпендикулярного $(\mathbf{B} \perp \mathbf{Z}$, нижние стрелки) центров ${ }^{151} \mathrm{Eu}^{2+}$ в $\mathrm{Y}_{3} \mathrm{Al}_{5} \mathrm{O}_{12}$ при $300 \mathrm{~K}$ и $\mathbf{B} \| \mathbf{C}_{2}$. Меньшая пиковая интенсивность сигналов параллельного центра обусловлена бо́льшим перекрытием компонент CTC. 
нами к центру с $\mathbf{B}\|\mathbf{Z}\| \mathbf{C}_{2} \|\langle 110\rangle$, ось $\mathbf{Y}$ параллельна ортогональному направлению $\langle 110\rangle$, ось $\mathbf{X} \|\langle 100\rangle$.

Спектры ЭПР кристаллов, легированных кремнием и гафнием, характеризуются переходами $\mathrm{Eu}^{2+} \mathrm{c}$ идентичными положениями, но различаются интенсивностью сигналов; в кристаллах с кремнием интенсивность ромбических центров европия примерно в 2 раза больше, чем в кристаллах с гафнием. Также кристаллы с кремнием демонстрируют дополнительные переходы $\mathrm{Eu}^{2+}$, которые не описываются в симметрии $D_{2}$. Поскольку замещение ионами европия в гранате ионов алюминия крайне маловероятно, скорее всего, это переходы центров $\mathrm{Eu}^{2+}$, локализованных в позиции иттрия, имеющих близко расположенный зарядокомпенсирующий дефект (например, четырехзарядный ион кремния в позиции $\mathrm{Al}^{3+}$, расположенной на расстоянии $\sim 0.3 \mathrm{~nm}$ вдоль $X$ ) и более низкую симметрию. Можно предполагать, что указанные эффекты связаны с большой разницей ионных радиусов $\mathrm{Si}^{4+}(0.04 \mathrm{~nm})$ и $\mathrm{Hf}^{4+}(0.071 \mathrm{~nm})$ и их локализацией в различных структурных позициях. Кроме того, было замечено, что в кристаллах с кремнием интенсивность сигналов ионов $\mathrm{Mo}^{3+}$ и $\mathrm{Cr}^{3+}$, присутствующих в образцах в качестве неконтролируемых примесей, примерно в 5 раз больше, чем в кристаллах с гафнием.

Спектр центров ${ }^{151} \mathrm{Eu}^{2+}$ описывался спиновым гамильтонианом симметрии $D_{2}$ в определении авторов [6]

$$
\begin{aligned}
H_{\mathrm{sp}}= & g \beta(\mathbf{B S})+1 / 3\left(b_{20} O_{20}+b_{22} O_{22}\right) \\
& +1 / 60\left(b_{40} O_{40}+b_{42} O_{42}+b_{44} O_{44}\right) \\
& +1 / 1260 \sum_{m} b_{6 m} O_{6 m}+A_{x} I_{x} S_{x}+A_{y} I_{y} S_{y}+A_{z} I_{z} S_{z}
\end{aligned}
$$

где $g-g$-фактор, $\beta$ - магнетон Бора, $O_{n m}-$ спиновые операторы Стивенса, $b_{n m}$ - параметры тонкой структуры, $I_{i}$ и $S_{i}$ - операторы проекций ядерного и электронного спинов ${ }^{151} \mathrm{Eu}^{2+}, A_{i}$ - параметры сверхтон-

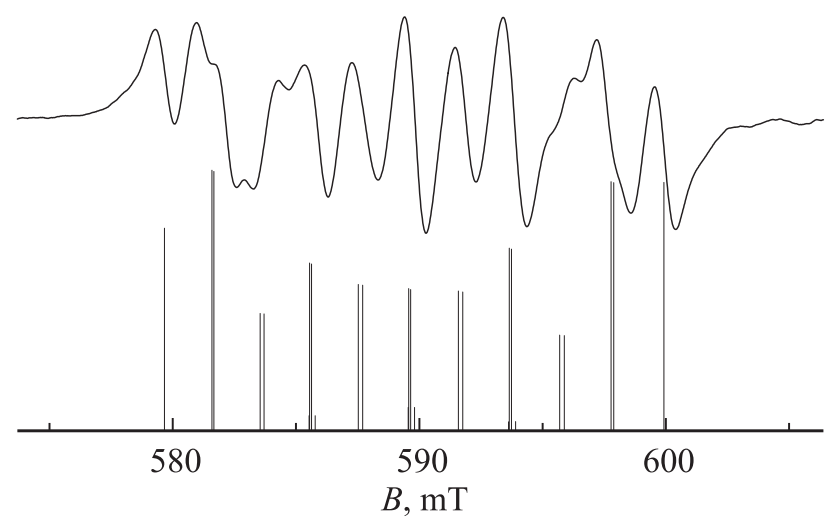

Pис. 4. ЭПР-спектр и расчетные положения компонент СТС четырех магнитно-эквивалентных центров ${ }^{151} \mathrm{Eu}^{2+}$ при $\mathbf{B} \| \mathbf{C}_{4}$ и $300 \mathrm{~K}$. Компоненты СТС представляют переходы с электронноядерных состояний 19-24 электронного уровня 4 в состояния 25-30 уровня 5 .
Параметры спинового гамильтониана и среднеквадратичные отклонения $F(N)$ расчетных частот от экспериментальных (где $N$ - число использованных экспериментальных положений электронно-ядерных переходов) центров ${ }^{151} \mathrm{Eu}^{2+}$ в $\mathrm{Y}_{3} \mathrm{Al}_{5} \mathrm{O}_{12}$ (все величины, кроме $g$-фактора, измеряются в $\mathrm{MHz}$ )

\begin{tabular}{c|c|c|c|c}
\hline Параметр & $120 \mathrm{~K}$ & $200 \mathrm{~K}$ & $300 \mathrm{~K}$ & $120 \mathrm{~K}[1]^{*}$ \\
\hline$g$ & $1.9905(7)$ & $1.9905(7)$ & $1.9905(7)$ & 1.991 \\
$b_{20}$ & $-8927(3)$ & $-8928(4)$ & $-8940(4)$ & 8930 \\
$b_{22}$ & $-8763(6)$ & $-8868(8)$ & $-9029(7)$ & 8762 \\
$b_{40}$ & $20(2)$ & $30(3)$ & $29(2)$ & -22 \\
$b_{42}$ & $-929(9)$ & $-952(10)$ & $-953(9)$ & 912 \\
$b_{44}$ & $-915(15)$ & $-838(18)$ & $-842(16)$ & 898 \\
$b_{60}$ & $-11(3)$ & $-9(3)$ & $-8(3)$ & - \\
$A_{x}=A_{y}=A_{z}$ & $-102(2)$ & $-102(2)$ & $-102(2)$ & 100 \\
$F(N)$ & $14(74)$ & $15(78)$ & $13(74)$ & $39(52)$
\end{tabular}

Пр и ме чан и е. * В работе [1] предполагается,что параметр $b_{20}>0$.

кого взаимодействия. Влияния ядерного квадрупольного взаимодействия на спектр ЭПР замечено не было.

В связи с тем, что в ряде случаев наблюдаемые компоненты СТС формируются электронно-ядерными переходами нескольких почти магнитно-эквивалентных (из-за неидеальности ориентации) центров (рис. 4), экспериментальные значения положений этих переходов оказываются приблизительными и не могут использоваться в процедуре оптимизации параметров спектра. В результате учета около 70 электронно-ядерных переходов при каждой температуре $(120,200$ и $300 \mathrm{~K}$, рис. 1) в пяти ориентациях магнитного поля методом наименьших квадратов получены параметры спинового гамильтониана, приведенные в таблице (о знаках параметров см. далее). Там же приведены параметры тонкой структуры из [1]. Остальные параметры шестого ранга не приводятся ввиду их слабого влияния на положения сигналов.

\section{4. Обсуждение результатов}

Знание параметров спинового гамильтониана позволяет обосновать соотнесение параллельного центра со спектром, характеризующимся менее разрешенной СТС (см. разд. 3 и рис. 1 и 3). Логично предполагать, что в данном случае уширение компонент спектра обусловлено статическим разбросом (флуктуациями) параметров гамильтониана. Если отклонения от среднего значения разных параметров независимы, то величины, пропорциональные второму моменту линии, можно оценить согласно выражению

$$
M_{2} \sim \sum_{n m}\left(\frac{d B_{\text {res }}}{d b_{n m}}\right)^{2}\left\langle\Delta b_{n m}^{2}\right\rangle,
$$

где $B_{\text {res }}-$ резонансное положение компоненты СТC, \langle\rangle - знак усреднения. Значения производных $d B_{\text {res }} / d b_{2 m}$ и $d B_{\text {res }} / d b_{4 m}$ имеют примерно одинаковую величину. Ввиду малости параметров четвертого ранга 

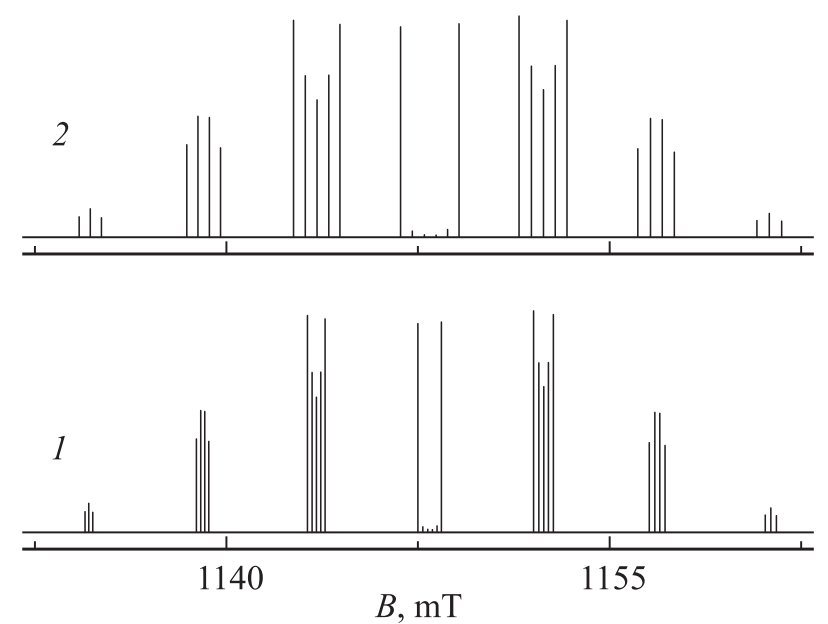

Рис. 5. Расчетные положения и интенсивности компонент СТС перехода $4 \leftrightarrow 5$ при В $\| \mathbf{C}_{4}$ и 300 К. $1-b_{20}<0, A<0$, $2-b_{20}>0, A<0$.

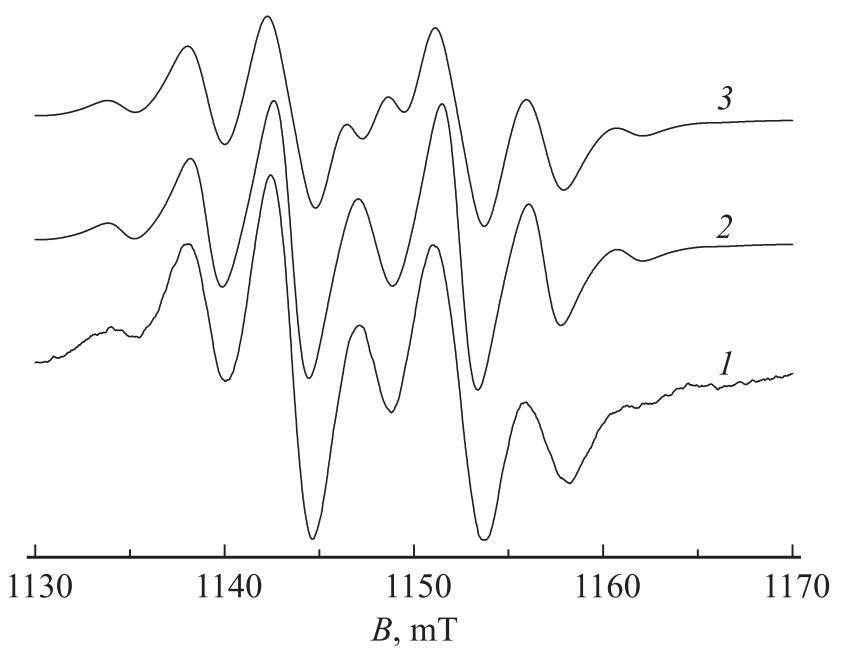

Рис. 6. Спектр ЭПР перехода $4 \leftrightarrow 5$ при $\mathbf{B} \| \mathbf{C}_{4}$ и $300 \mathrm{~K}$. 1 - эксперимент, 2 - расчет с $b_{20}<0, A<0,3-$ расчет c $b_{20}>0, A<0$. Форма индивидуальной линии - лоренциан с шириной между пиками первой производной $\Delta B_{p p}=1.8 \mathrm{mT}$.

их среднеквадратичные отклонения также должны быть малы. Поэтому в выражении (2) можно ограничиться только слагаемыми второго ранга. Расчет (переходы в поле $1400 \mathrm{mT}$ на рис. 1) дает (в $\mathrm{mT} / \mathrm{MHz}$ ) $d B_{\text {res }} / d b_{20}=0.144, d B_{\text {res }} / d b_{22}=0.009$ для параллельного центра и $d B_{\text {res }} / d b_{20}=0.054, d B_{\text {res }} / d b_{22}=0.081$ для перпендикулярного центра. Предполагая равенство $\left\langle\Delta b_{20}^{2}\right\rangle$ и $\left\langle\Delta b_{22}^{2}\right\rangle$ (поскольку $b_{22} \approx b_{20}$ ), получаем, что второй момент линии параллельного центра $(\mathbf{B} \| \mathbf{Z})$ более чем в 2 раза превышает $M_{2}$ сигнала перпендикулярного центра. Примерно такое же соотношение вторых моментов реализуется для двух пар электронных переходов, приведенных на рис. 3. Следовательно, соотнесение двух одиночных центров с наблюдаемыми спектрами при В $\| \mathbf{C}_{2}$ сделано верно.
В работе [1] абсолютные знаки параметров тонкой структуры ${ }^{151} \mathrm{Eu}^{2+}$ не определялись, при этом предполагалось, что $b_{20}>0$. Как видно, в таблице знаки параметров спинового гамильтониана центров ${ }^{151} \mathrm{Eu}^{2+}$ противоположны знакам, приведенным в [1]. Это связано с тем, что нам удалось найти переход, вид СТС которого заметно зависит от соотношения знаков начального расщепления и параметра сверхтонкого взаимодействия (рис. 5), и на этом основании определить знак $b_{20}$.

На рис. 6 представлена экспериментальная сверхтонкая структура электронного перехода $4 \leftrightarrow 5$, наблюдаемого при $\mathbf{B} \| \mathbf{C}_{4}$ и $300 \mathrm{~K}$ в поле $\sim 1150 \mathrm{mT}$, характер СТС в исследованном диапазоне температур $(120-300 \mathrm{~K})$ не изменяется. СТС этого перехода формируется четырьмя эквивалентными центрами $\mathrm{Eu}^{2+}$, для которых реализуется ориентация магнитного поля $\theta=45^{\circ}, \varphi=90^{\circ}$.

Также на рис. 6 приведены результаты симуляции спектра с одинаковыми и противоположными знаками параметров $b_{20}$ и $A$. При симуляции данного спектра считалось, что форма индивидуальной линии - лоренциан с шириной между экстремумами первой производной $\Delta B_{p p}=1.8 \mathrm{mT}$. В предположении гауссовой формы линии процедура симуляции дает близкие результаты. Согласно данным работ [7-9], знак параметра сверхтонкого взаимодействия отрицательный. Очевидно (рис. 6), что адекватное описание спектра дает расчет с $b_{20}<0$, $A<0$. Неидеальное согласие экспериментального и симулированного спектров, возможно, обусловлено неучетом ядерного квадрупольного взаимодействия. Между уровнями 4 и 5 четырех эквивалентных центров при $\mathbf{B} \| \mathbf{C}_{4}$ происходит несколько переходов. СТС одного из них, наблюдаемого в поле $590 \mathrm{mT}$, показана на рис. 4 . Однако изменение соотношения знаков параметров тонкой и сверхтонкой структур практически не влияет на вид расчетного спектра этого перехода, так же как и всех других исследованных сигналов, кроме описанного выше.

\section{5. Заключение}

Выращены качественные монокристаллы иттрийалюминиевого граната с примесью европия, обогащенного изотопом ${ }^{151} \mathrm{Eu}$, и четырехвалентных ионов кремния или гафния, обеспечивающих существование центров $\mathrm{Eu}^{2+}$.

В трех ориентациях магнитного поля (B $\left\|\mathbf{C}_{2}, \mathbf{B}\right\| \mathbf{C}_{4}$, $\mathbf{B} \| \mathbf{C}_{3}$ ) исследованы температурные и частотные зависимости положений центров ${ }^{151} \mathrm{Eu}^{2+}$. Путем оптимизации методом наименьших квадратов определены параметры ромбического спинового гамильтониана при 120, 200 и $300 \mathrm{~K}$. В результате сравнения экспериментального и расчетного характера сверхтонкой структуры одного из переходов при $\mathbf{B} \| \mathbf{C}_{4}$ и $300 \mathrm{~K}$ определены относительные знаки начального расщепления и параметра СТВ.

Авторы благодарны А.Д. Горлову и М.Ю. Артемову за полезные обсуждения работы. 


\section{Список литературы}

[1] В.А. Важенин, А.П. Потапов, Г.Р. Асатрян, Ю.А. Успенская, А.Г. Петросян, А.В. Фокин. ФТТ 58, 1573 (2016).

[2] А.А. Чернов, Е.И. Гиваргизов, Х.С. Багдасаров, В.А. Кузнецов, Л.Н. Демьянец, А.Н. Лобачев. Современная кристаллография. Наука, М. (1980). 337 с.

[3] A.G. Petrosyan. J. Cryst. Growth 139, 372 (1994).

[4] Т.И. Бутаева, А.Г. Петросян, А.К. Петросян. Неорган. материалы 24, 430 (1988).

[5] R.D. Shannon. Acta Cryst. A 32, 751 (1976).

[6] С.А. Альтшулер, Б.М. Козырев. Электронный парамагнитный резонанс. Наука, М. (1972). С. 121.

[7] J. Bronstein, V. Volterra. Phys. Rev. 137, A1201 (1965).

[8] J.S.M. Harvey, H. Kiefte. Can. J. Phys. 47, 1505 (1969).

[9] А.Д. Горлов. ФТТ 56, 2115 (2014). 